\title{
HIERARCHICAL SEGMENTATION OF VEGETATION AREAS IN HIGH SPATIAL RESOLUTION IMAGES BY FUSION OF MULTISPECTRAL INFORMATION
}

\author{
Felipe Calderero $^{1}$, Ferran Marqués ${ }^{1}$, Javier Marcello ${ }^{2}$, Francisco Eugenio ${ }^{2}$ \\ ${ }^{1}$ Signal Theory and Communications Department. Technical University of Catalonia (UPC) \\ E-mail: felipe.calderero@upc.edu, ferran.marques@upc.edu \\ ${ }^{2}$ Signals and Communications Department. University of Las Palmas of Gran Canaria \\ E-mail: fmarcello@dsc.ulpgc.es, feugenio@dsc.ulpgc.es
}

\begin{abstract}
A new region-based methodology for the automated extraction and hierarchical segmentation of vegetation areas into high spatial resolution images is proposed. This approach is based on the iterative and cooperative fusion of the independent segmentation results of equal or different resolution spectral bands, combined with an unsupervised classification into vegetation and novegetation regions. The result is a hierarchy of partitions with most relevant information at different levels of resolution of the vegetation areas. In addition, the high flexibility of the scheme allows different configurations depending on the final purpose. For instance, considering the size of the vegetation areas into the hierarchy, or prioritizing the information into the high resolution panchromatic band to improve the accuracy of both vegetation extraction and segmentation. This general tool for vegetation analysis is tested into high spatial resolution images from IKONOS and QuickBird satellites.
\end{abstract}

Index Terms - Image segmentation, region merging, multispectral images, information fusion

\section{INTRODUCTION}

High spatial resolution imagery offers new opportunities for potentially more accurate detection and classification than traditional satellite imagery. Nevertheless, an increasingly smaller spatial resolution causes that single pixels no longer capture the characteristics of classification target. Hence, an increasingly smaller spatial resolution does not necessarily benefit classification performance and accuracy for traditional pixel-based classification approaches [1]. Thus, preliminary feature extraction techniques are of great importance for the success of classification methodologies when applied to high resolution imagery.

One important feature extraction approach is image segmentation. It partitions the image into a set of homogeneous regions under a certain criterion. Regions are a first level of abstraction, being more robust and semantically meaningful than pixels. A large number of algorithms have been proposed for image segmentation [2] but there has been little progress in the unsupervised segmentation of multispectral imagery [3-5]. These approaches do not consider the fact that the required level of detail into the segmentation depends on the final application, especially for vegetation characterization. Hence, a general tool for vegetation analysis should provide a hierarchical representation where, instead of a unique partition, different region-based explanations at different levels of detail are given. In addition, most of the previous techniques assume equal resolution bands and do not consider including higher resolution information, such as the panchromatic band. In general, the higher resolution information is included into the low resolution bands by an enhancement process based on image fusion techniques [6] at pixel level. However, it is not clear how image fusion influences the next steps into the processing chain, such as segmentation and classification.

In this context we present a new region-based methodology for the automated extraction and hierarchical segmentation of high spatial resolution images. Although the proposed technique may be applied to the study of different types of information, this work focuses on the extraction and segmentation of the vegetation areas. This approach is based on a high level iterative and cooperative scheme that fuses the independent segmentation results of each spectral band and an unsupervised vegetation and no-vegetation classification. This strategy provides an unsupervised hierarchical segmentation of the vegetation areas of the image. The high flexibility of the proposed scheme allows obtaining:

- An unsupervised hierarchical vegetation segmentation for equal resolution bands (for instance, B, G, R, IR) including or not scale information into the hierarchy.

- An unsupervised hierarchical vegetation segmentation for different resolution bands (for instance, including panchromatic information), improving the accuracy and resolution of both vegetation extraction and segmentation.

The proposed framework has been tested for high spatial resolution images from commercial satellites, such as IKONOS and QuickBird. The paper is structured as follows. Section 2 presents the general cooperative region merging scheme applied to the hierarchical multispectral segmentation. Section 3 presents the experimental results for the segmentation of equal resolution bands (Section 3.1 ), and including the higher resolution panchromatic band (Section 3.2). Finally, conclusions are outlined in Section 4.

\section{MULTISPECTRAL COOPERATIVE REGION MERGING}

The general cooperative region merging strategy for multispectral hierarchical segmentation is presented in Figure 1. It is formed by five main steps: the region merging step, where the separated segmentation for each spectral band is performed; the segmentation fusion step, where the independent segmentation results of each band are combined into a consensus partition; the region-based classification step, where an unsupervised vegetation classification of the regions is performed; the meet step, where the classification and segmentation results are fused; and the scale filter, where the scale consistency of the partitions is assured. 
The central idea is to let the system evolve by itself, starting with a basic agreement (given by an initial partition) and searching for partitions with decreasing number of regions by further consensus iteration after iteration. This is done instead of finding a coarser direct consensus partition for spectral band partitions (risking introducing under-segmentation errors). It can be shown that this iterative scheme provides with a partition hierarchy: iteration after iteration, a partition coarser than the previous partition is obtained. This scheme was successfully used into the joint segmentation of other types of information, such as color and depth information [7].

\subsection{Region Merging Step}

A region merging step is associated with each criteria or information source. Starting from an initial partition of the image data (or directly all pixels at the first iteration), this step performs a region merging process providing a merging sequence of the regions in the initial partition according to the characteristics of the spectral band considered (see Figure 1).

The region merging techniques used in this work are based on a modified version of the general region merging techniques based on information theory statistical measures proposed in [8] (here using the ASH probability density function estimator [9]). Precisely, a merging process formed by a Bhattacharyya merging criterion and a scale-based merging order is chosen for its good compromise between under- and over-segmentation errors, in the context of both color homogeneous and texture region segmentation.

\subsection{Segmentation Fusion: Maximum Mutual Information Partition}

The goal of this block is to determine a good consensus partition among the independent segmentation result of each band. In other words, observing the sequence of mergings we would like to determine the set of region fusions where the independent processes mostly agree. Hence, we search for the partition with smallest number of regions where the segmentation results of each band still have a large degree of agreement. This process is done in two steps: (i) creating a fused merging sequence among the different segmentation results; and (ii) determining the partition within the fused merging sequence with smallest number of regions that still shows a large degree of agreement with respect to the segmentation of the individual spectral bands.

\subsubsection{Creation of a fused merging sequence}

To have a common reference, the merging sequences are written in terms of the removal of the boundaries between regions of the initial partition. For instance, removing boundary $(\mathrm{A}, \mathrm{B})$ is equivalent to merging regions $\mathrm{A}$ and $\mathrm{B}$ of the initial partition. The fused merging sequence is then a new boundary removal ordering obtained after combining the orderings provided by the region merging process of each band. Common combination functions are the maximum (preventing undersegmentation), minimum (preventing oversegmentation), or mean (compromise).

\subsubsection{Determining the multispectral consensus partition}

Once the fused merging sequence has been computed, the next step is to determine the segment of mergings where segmentation results in each band present a significant agreement. The partition at this point will be considered as the consensus partition among the different spectral bands.

To study the evolution of the agreement into the merging process, the difference in priority given by each individual band and the

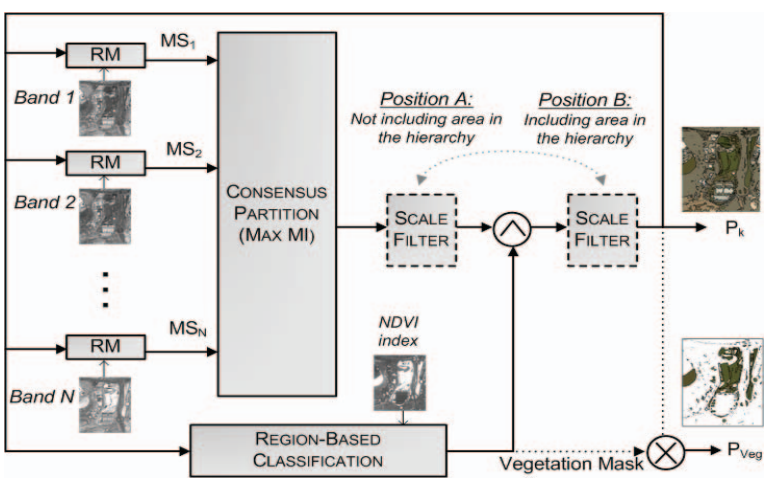

Figure 1. Cooperative region merging scheme for multispectral hierarchical vegetation extraction and segmentation.

new fused sequence is computed and ordered according to the fused sequence. In general, the differences in priority are small for the first region mergings (merging decisions are clear at this stage). Nevertheless, as the merging process continues, the differences increase as regions present more complex models and depend on previous decisions. Hence, the initial correlation of the different sequences decreases and, at some point, they significantly diverge. We model this behavior as follows. Consider the merging sequence of a spectral band, $X=\left\{x_{1}, \ldots, x_{n}\right\}$, and the fused sequence, $Y=\left\{y_{1}, \ldots, y_{n}\right\}$ obtained combining the merging sequences of the multispectral bands (as explained in Section 2.2.1). We expect that both sequences have a significant correlation at an initial segment that is not present after this point (both sequences can be considered independent). Under these premises, their mutual information is given by $I(X, Y)=H(X)-H(X \mid Y)$, where the conditional entropy can be written as:

$$
H(X \mid Y)=H\left(x_{1}, \ldots, x_{k} \mid y_{1}, \ldots, y_{k}\right)+H\left(x_{k+1}, \ldots, x_{n}\right)
$$

The first term on the right represents the conditional entropy in the part where both sequences are correlated, and the second refers to the segment where they significantly differ.

To compute the first term we assume that the difference between the merging sequence of a band and the fused sequence in the part where both sequences are similar can be considered as independent samples of a certain random variable, $e$. In turn, the second term is determined assuming that the probability of all mergings at a certain stage is equally likely (which may be true assuming no knowledge on the merging processes carried in each band), and assuming that the order given to each element is independent of the previous mergings (which is a simplification, since larger regions will be formed by the merging of elementary regions, as thus, may partially depend on previous fusions). Thus,

$$
H(X \mid Y) \approx k \cdot H(e)+\sum_{j=k+1}^{n} \log (n-j)
$$

In this context, finding the initial segment where both sequences mostly agree is equivalent to determine the value of $k$ that maximizes the mutual information. Using the same assumption to compute the entropy of the merging sequence, $H(X)$, as before, we obtain:

$$
k^{*}=\arg \max _{k} I(X \mid Y)=\arg \max _{k}\left[\sum_{j=1}^{k} \log (n-j)-k \cdot H(e)\right]
$$


This expression can be extended to the set of merging sequences obtained from each spectral band:

$$
k^{*}=\arg \max _{k} I\left(X_{1}, \ldots, X_{M} \mid Y\right)
$$

where

$$
I\left(X_{1}, \ldots, X_{M} ; Y_{k}\right)=\sum_{i=1}^{M} I\left(X_{i} ; Y\right)
$$

considering merging sequences independent between them. Finally, multispectral consensus partition is obtained, using a conservative approach, by the removal of the first $0.75 \cdot k^{*}$ initial boundaries.

\subsection{Region-Based Classification}

This block performs an unsupervised classification into vegetation and no-vegetation of the regions into the initial partition at the current iteration, using the information provided by the normalized differential vegetation index (NDVI) [10]. For each region, the NDVI mean value over all its pixels is determined. Then, regions are classified based on their mean NVDI index into two classes using k-means or fuzzy k-means algorithm. The vegetation partition that is fused with the multispectral consensus partition (see Section 2.5) is obtained by relabeling the vegetation classification.

\subsection{Meet Step}

The meet operation outputs the intersection of the input partitions, that is, a partition that includes all boundaries present in the input partitions. In other words, pixels with equal label vectors (each component being the label at a particular band) belong to the same region into the meet partition.

The goal of this block is to assure that the relevant information about the vegetation and no-vegetation areas is preserved into the consensus partition of the multispectral bands. In that sense, the vegetation partition is included as prioritary information into the system, preventing vegetation and no-vegetation areas to merge.

\subsection{Scale Filter}

The scale filter assures the scale consistency of all the obtained partitions, that is, removes the set of regions that are too small to be significant at the current level of resolution. For that purpose an adaptive scale threshold is defined on the region areas, similarly to the scale-based merging order used in [8]:

$$
T_{\text {scale }}=\alpha \cdot \frac{\text { Image Area }}{\text { Number of Regions }}
$$

The $\alpha$ parameter controls the minimum resolution at each scale. In our experiments, we have chosen a low value for this parameter, $\alpha=0.07$, to be sure that only clearly meaningless regions at that scale are discarded. Out-of-scale regions are merged according to the most reliable or relevant available information. For instance, for equal resolution channels NVDI is used (see Section 3.1) and so is the panchromatic band for the different channel resolution configuration (see Section 3.2). As the scale-filtered partition is a coarser partition that the previous one, the hierarchical structure of the output partitions is maintained.

As shown in Figure 1, the position of the scale filter determines whether the area of the vegetation is introduced or not into the created hierarchy. If the filter is placed before the meet operation (Position A), isolated vegetation areas are preserved into the hierarchical segmentation, independently of their size. On the contrary, if the scale filter is located after the meet operation (Position B), only the vegetation areas that are large enough to be significant at the given scale are preserved.

\section{EXPERIMENTAL RESULTS}

The performance of the proposed framework has been tested for high spatial resolution images from commercial satellites, such as IKONOS (four channels of multispectral data at $4 \mathrm{~m}$ resolution and one panchromatic channel at $1 \mathrm{~m}$ resolution) and QuickBird (four channels of multispectral data with $2.4 \mathrm{~m}$ resolution and one panchromatic channel with $0.6 \mathrm{~m}$ resolution).

\subsection{Configuration for Equal Resolution Bands}

In this first case, four different bands with equal resolution $(B, G$, $\mathrm{R}, \mathrm{IR})$ were considered for each multispectral image. As shown in Figure 1, each band was injected to a different region merging process. NDVI was computed and used as input for the regionbased classification block. Independently of its position, the scale filter used the NDVI to merged out-of-scale regions.

In Figure 2, an example of the hierarchical segmentation results obtained on a $221 \times 261$ subimage of a Quickbird multispectral image is shown. In this case, the maximum of the merging sequences was used to create the consensus partition among the different segmentations of each band. We observed that the results are similar for the maximum and mean functions, and significantly worst for the minimum. In the presented example, the scale filter is set in Position A (see Fig. 1) such that the vegetation area is not included into the partition hierarchy. The same configuration but including the area into the hierarchy is shown in Figure 3 for the same subimage. Note that in this case, as the level of detail into the hierarchy decreases, only the vegetation areas with enough area to be relevant at the given scale are preserved.

Applications analyzing vegetation areas independently of their size, and hence, interesting on preserving even small vegetation areas will find into the first configuration a valuable solution. Moreover, applications searching for vegetation areas of a certain size, or interested on the granularity and distribution of the green areas, will prefer the second configuration.

\subsection{Configuration for Different Resolution Bands}

In this case, in addition to the four equal resolution bands used in the previous experiments (B, G, R, IR), the panchromatic band was also used. To provide with partitions of equal resolution the four bands of equal resolution were interpolated by a factor of four using nearest neighbor interpolation algorithm. Instead of the NDVI, the panchromatic band was used by the scale filter (independently of its position) to merge out-of-scale regions, as it is considered as the most accurate channels thanks to its higher spatial resolution.

An example for a subset of $201 \times 146$ pixels into the low resolution bands $(804 \times 584$ pixels at the panchromatic band) image is shown in Figure 4. The hierarchical segmentation using only the equal resolution bands and including also the panchromatic band were computed. In both cases, the maximum function was used for the combination, and the vegetation area was not included into the hierarchy. From the two levels of the partition hierarchy shown, it can be seen that the use of the panchromatic information improves the correct segmentation of the field structures (that is more evident at the partition with higher level of detail), and also is able to improve the classification into vegetation and no-vegetation 

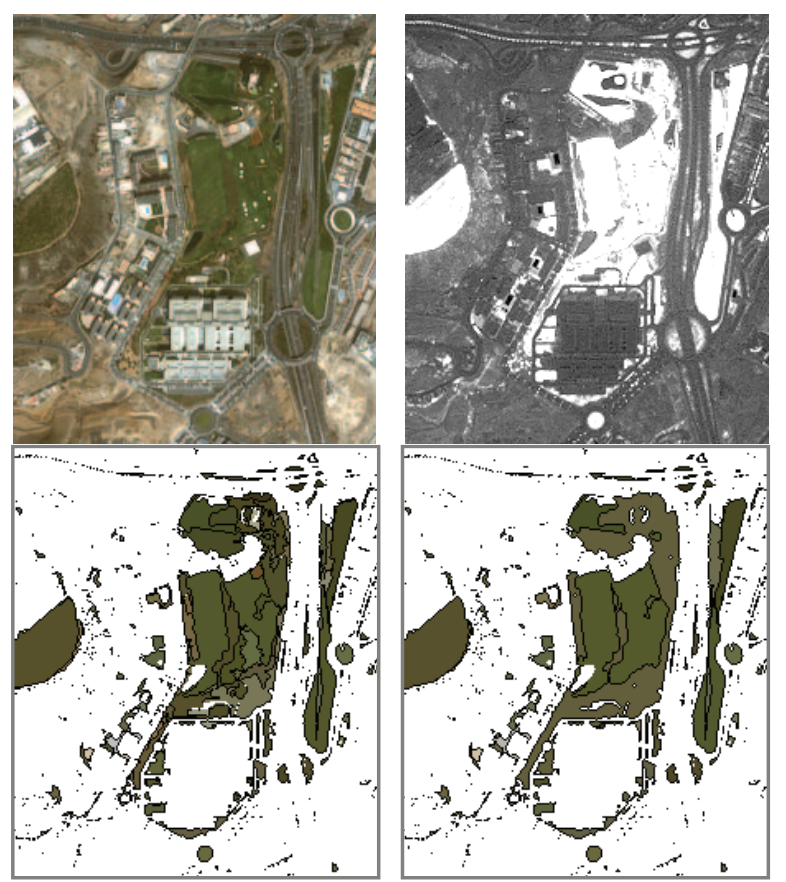

Figure 2. Hierarchical vegetation segmentation of a Quickbird subimage. First row. Left: Portion of original multispectral image (R,G,B composition). Righ: NDVI index. Second row: Two different levels of the hierarchy of partitions. Example computed using maximum fusion criteria and not including the vegetation area into the hierarchy (Position A). No-vegetation areas are in white.

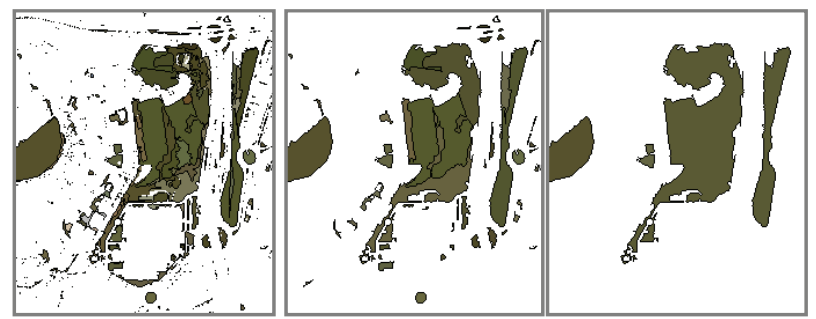

Figure 3. Example of different levels of segmentation obtained including the vegetation area into the creation of the hierarchy (Position B), using the same image as in Figure 2.

areas (more evident at the partition with less level of detail). Thus, the previous results show that including more accurate spatial information (panchromatic band) not only improves the segmentation of the multispectral images, but also the classification performance into vegetation and no-vegetation areas. This is a consequence of the cooperation between the segmentation and classification stages proposed in our scheme.

\section{CONCLUSIONS}

The presented results show that the proposed hierarchical multichannel segmentation approach provides an unsupervised and flexible tool for the analysis and classification of vegetation into a broad range of remote sensing applications.

\section{REFERENCES}

[1] Q. Yu, P. Gong, N. Clinton, G. Biging, M. Kelly, and D. Schirokauer, "Object based detailed vegetation classification with airborne high spatial

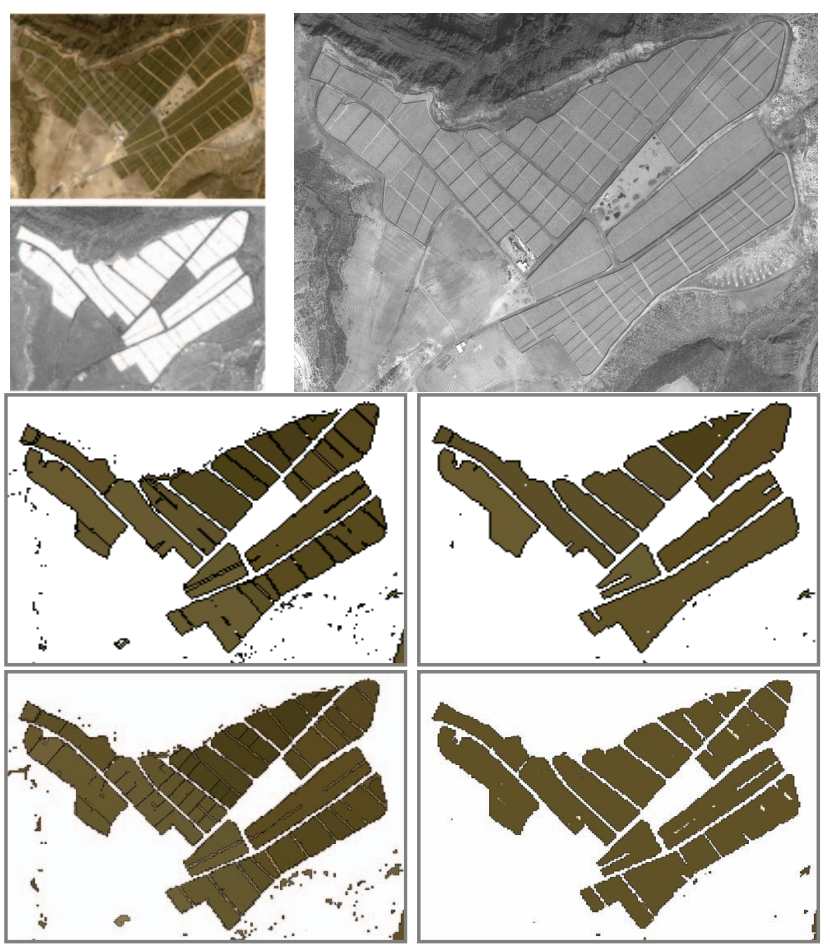

Figure 4. Hierarchical vegetation segmentation of Quickbird subimage. The first row shows the color (R,G,B composition) image (top-left), the NDVI index (bottom-left), and the panchromatic band (right) (not at their proportional scale). Second row shows the results for two different levels of the partition hierarchy using B, G, R, and IR bands (equal resolution channels) in the segmentation process. The third row shows partitions at similar levels of the hierarchy for the scheme configuration including also the panchromatic band.

resolution remote sensing imagery," Photogrammetric Engineering and Remote Sensing, vol. 72, pp. 799-811, 2006.

[2] N. Pal and S. Pal, "A review on image segmentation techniques," Pattern Recognition, vol. 26, pp. 1277-1294, 1996.

[3] P. Li and X. Xiao, "Multispectral image segmentation by a multichannel watershed-based approach," International Journal of Remote Sensing, vol. 28, no. 19, pp. 4429-4452, 2007.

[4] M. Sellami, F. Chaabane, C. Fetita, "Morphological Segmentation of Multispectral Images for Land Cover Mapping," Proc. of IGARSS 2008, vol.3, pp. 326-329, 2008.

[5] Y. Zhang, X. Feng, and X. Le, "Segmentation on Multispectral Remote Sensing Image Using Watershed Transformation," Proc. of CISP '08, vol.4, pp.773-777, 2008.

[6] F. Laporterie-Dejean, H. de Boissezon, G. Flouzat, M.-J. LefevreFonollosa, "Thematic and statistical evaluations of five panchromatic/multispectral fusion methods on simulated PLEIADESHR images," Information Fusion, vol. 6, no. 3, pp. 193-212, 2004.

[7] F. Calderero and F. Marques, "Hierarchical fusion of color and depth information at partition level by cooperative region merging," to appear in Proceedings of ICASSP'09, 2009.

[8] F. Calderero and F. Marques, "General region merging approaches based on information theory statistical measures," Proceedings of ICIP’08, pp. 3016-3019, 2008.

[9] D. W. Scott, "Averaged shifted histograms: Effective nonparametric density estimators in several dimensions," The Annals of Statistics, vol. 13, no. 3, pp. 1024-1040, 1985.

[10] R. A. Schowengerdt, Remote sensing models and methods for image processing, Academic Press, 3rd edition, 2007. 